\title{
The Future of Nuclear Medicine as an Independent Specialty
}

\author{
Johannes Czernin, Ida Sonni, Aria Razmaria, and Jeremie Calais \\ Ahmanson Translational Theranostics Division, Department of Molecular and Medical Pharmacology, David Geffen School of \\ Medicine, UCLA, Los Angeles, California
}

In this article, we provide an overview of established and emerging conventional nuclear medicine and PET imaging biomarkers, as the diagnostic nuclear medicine portfolio is rapidly expanding. Next, we review briefly nuclear theranostic approaches that have already entered or are about to enter clinical routine. Using some approximations and taking into account emerging applications, we also provide some simplified business forecasts for nuclear theranostics. We argue that an optimistic outlook by the nuclear medicine community is crucial to the growth of the specialty and emphasize the urgent need for training adaptations.

Key Words: nuclear medicine; theranostics; molecular imaging

J Nucl Med 2019; 60:3S-12S

DOI: 10.2967/jnumed.118.220558

$\mathbf{T}$

he Accreditation Council for Graduate Medical Education lists 42 dedicated nuclear medicine programs in the United States (1), as opposed to 94 radiation oncology and $>200$ diagnostic radiology programs (2). Nuclear medicine has seen a decline of greater than $50 \%$ in Accreditation Council for Graduate Medical Education-accredited U.S. residency programs since 1990 and a $25 \%$ decrease compared with 2007-2008 (2). There is also a striking difference in "on-duty residents" in these specialties, with diagnostic radiology counting 4,697 trainees, radiation oncology containing 775 trainees, and nuclear medicine containing only 79 residents in 2017 (3). This declining trend over the past $20 \mathrm{y}$ was unexpected when put in the context of the significant advancements and innovations in molecular imaging and associated technologies during the same time frame.

PET/CT was clinically introduced in the early 2000s $(4,5)$. The Centers for Medicare \& Medicaid Services reimbursement became broad between 2005 and 2010. Glucose metabolism PET imaging for cancer evaluations (6) as well as for the assessments of cerebral function (7) and myocardial viability (8) is a key modality in oncology, neurology, and cardiology, respectively (9). Because of its great clinical and economic successes, PET/CT became an attractive diagnostic tool for radiologists. After only 4 mo of training, radiologists are authorized to interpret PET and all other diagnostic nuclear medicine studies. Training deficiencies are obvious and have been reported (10). The clinical

Received May 4, 2019; revision accepted Jul. 6, 2019.

For correspondence or reprints contact: Johannes Czernin, Ahmanson Translational Theranostics Division, Department of Molecular and Medical Pharmacology, David Geffen School of Medicine, UCLA, 10833 Le Conte Ave., Los Angeles CA 90095-7370.

E-mail: jczernin@mednet.ucla.edu

COPYRIGHT (C 2019 by the Society of Nuclear Medicine and Molecular Imaging. success of PET/CT resulted in an increase in nuclear radiology combined training programs from 0 in $1990-1991$ to 17 in 20172018.

Cardiac rest/stress SPECT imaging became the standard of care for diagnosis and prognostication for patients with coronary artery disease (11), and since 1996 the Certification Board of Nuclear Cardiology (CBNC), recognized by the U.S. Nuclear Regulatory Commission in 2001, has accredited more than 5,000 nuclear cardiologists. Nuclear cardiology became a profitable business, and private cardiology offices performed close to 8 million cardiac SPECT studies in 2011 (12).

The decline in dedicated nuclear medicine training programs in the United States is explained by a loss of ownership in technologies; minimal specific nuclear medicine training requirements in the United States (4 mo of training for radiologists); and professional compensation models, such as relative value units, that are not applicable and that threaten the financial viability of nuclear medicine. Relative value units are a value metric used in the U.S. Medicare reimbursement system to assign monetary value for specific physician services. These do not account for the low patient volumes and long study durations that are the rule in nuclear medicine.

Recently, the outlook for nuclear medicine has markedly improved. Radionuclide imaging assays diagnose and phenotype disease, provide prognostic information, survey the whole body for target expression, determine drug-target interactions, and assess treatment responses early after therapy initiation (see the discussion of imaging biomarkers later in this article). Nuclear theranostics (the use of radionuclide pairs for targeted molecular imaging and molecular radiotherapy) is becoming an important component of cancer care. As an example, targeting somatostatin receptors diagnostically and therapeutically is now a clinically established and reimbursed approach in the management of neuroendocrine tumors (NETs) $(13,14)$. More recently, ${ }^{123} \mathrm{I}$-iobenguane and ${ }^{131} \mathrm{I}$-iobenguane were approved for targeting the norepinephrine reuptake transporter in neuroblastoma and paraganglioma (15).

Importantly, various approaches to targeting prostate-specific membrane antigen (PSMA) diagnostically and therapeutically have been deployed clinically in many countries (16). This list will certainly grow over the next 5-10 y.

The application of these diagnostic assays and therapeutic approaches to the greatest benefit of patients requires profound knowledge and understanding, which in turn must be based on extensive training and learning.

In this article, we use SWOC (strengths, weaknesses, opportunities, and challenges) analysis, an approach commonly used in business to evaluate the internal and external factors influencing a company's success and growth, to separately describe the strengths, weaknesses, opportunities, and challenges facing nuclear medicine in the United States (Fig. 1). 


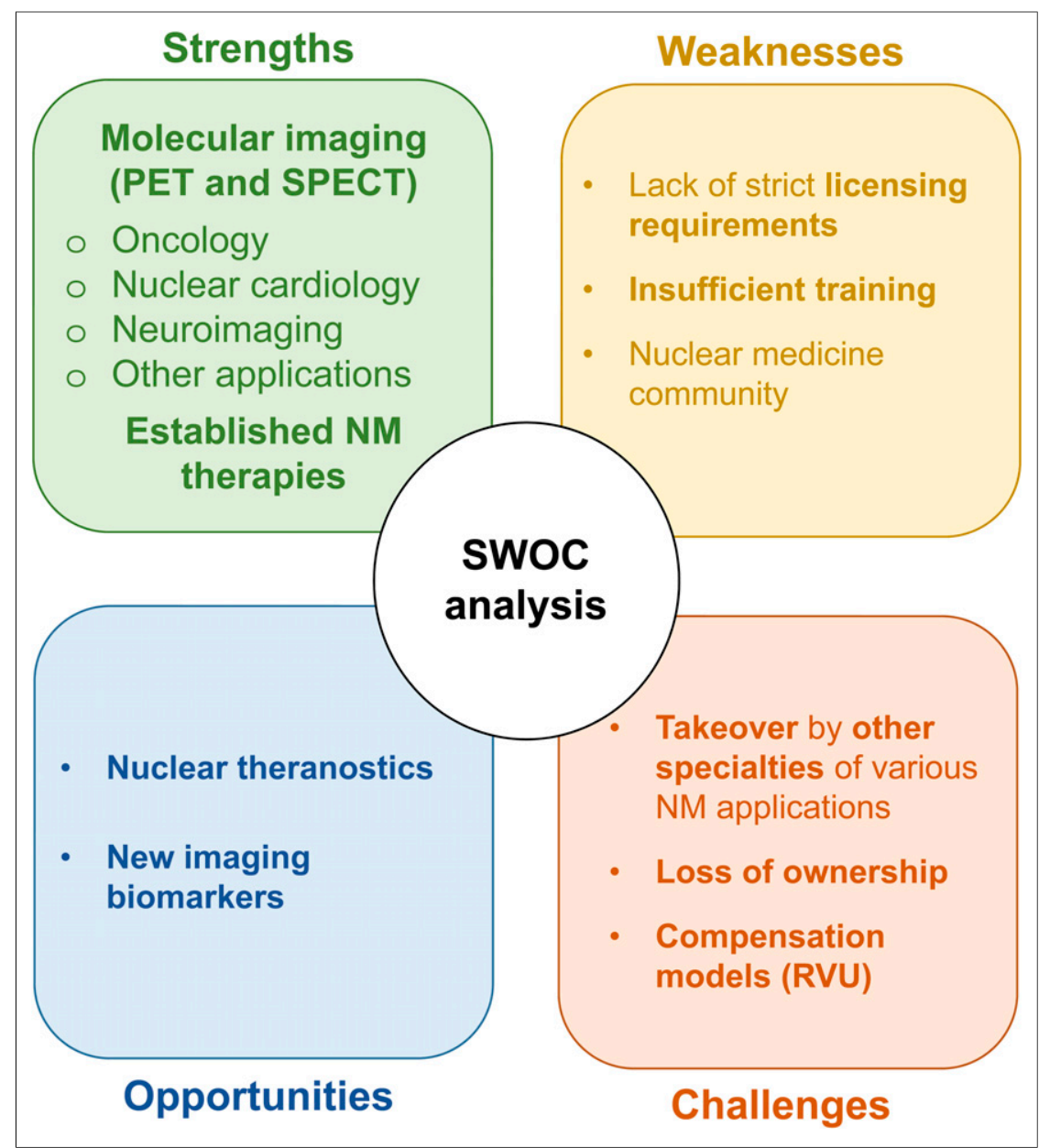

FIGURE 1. Schematic representation of SWOC (strengths, weaknesses, opportunities, and challenges) analysis. $\mathrm{NM}=$ nuclear medicine; $\mathrm{RVU}=$ relative value unit.

First, we provide an overview of established and emerging conventional nuclear medicine and PET imaging biomarkers, as the diagnostic nuclear medicine portfolio is rapidly expanding (strengths). Next, we review briefly nuclear theranostic approaches that have already entered or are about to enter clinical routine. Using some approximations and taking into account emerging applications, we also provide some simplified business forecasts for nuclear theranostics (opportunities). We argue that an optimistic outlook by the nuclear medicine community is crucial to the growth of the specialty. We emphasize the urgent need for training adaptations (challenges and weaknesses).

\section{STRENGTHS}

Many conventional nuclear medicine and PET imaging probes have been deployed for research and clinical applications (17). Their utility depends on their ability to answer various clinical questions, as highlighted in Table 1. Imaging biomarkers include small molecules, peptides and peptidomimetics, and antibodies and antibody fragments (18-21). Small molecules-metabolic and cell surface receptor-targeted probes-can successfully improve the management and outcome of patients affected by neurodegenerative (22), cardiovascular (23), inflammatory and infectious diseases (24), and cancer $(4,5)$.

\section{Established Imaging Biomarkers}

${ }^{18}$ F-FDG PET/CT imaging is a key imaging modality in cancer, and its use is still increasing. It remains the most successfully translated molecular imaging platform, a hybrid imaging tool for precision medicine. As stated by Buck et al., " Since its introduction in clinical medicine in 2001, PET/CT has represented one of the diagnostic modalities with the largest growth worldwide" (25). In the United States, it was performed in more than 2 million patients in 2018, a 7\% increase compared with 2017 (26). More than 5 million studies are performed worldwide each year. ${ }^{18} \mathrm{~F}-\mathrm{FDG}$ serves as a diagnostic and prognostic biomarker from early- to late-stage cancer. Its use as an intermediate endpoint biomarker for early therapy response assessments has changed the practice of oncology.

In the latest update of the European Society of Cardiology guidelines for the management of infective endocarditis and cardiac implantable electronic device infections, radiolabeled white blood cell SPECT/CT and ${ }^{18}$ F-FDG PET/CT were included.

In a remarkable shift, Jack et al. recently suggested that diagnoses of neurodegenerative diseases should no longer be "based on the clinical consequences of the disease (i.e., symptoms/signs)" but rather should be based on in vivo biomarkers, including those used in PET imaging (27). This paradigm shift provides more evidence that image-based biomarkers are gaining acceptance clinically and are indispensable for meaningful research.

${ }^{123}$ I-ioflupane (DaTscan; GE Healthcare) is used routinely for imaging of the dopaminergic system in patients with movement disorders (28). Table 2 shows a selection of U.S. Food and Drug Administration (FDA)-approved imaging biomarkers.

Myocardial viability assessment with ${ }^{18} \mathrm{~F}$-FDG PET stratifies patients toward revascularization versus cardiac transplantation. A strong glycolytic phenotype of hypoperfused myocardium is associated with functional recovery after revascularization (phenotypic biomarker) (29).

Glucose metabolic imaging serves as an intermediate endpoint biomarker for identifying patients with favorable treatment responses across all cancers and most therapies. Early and accurate treatment response assessments, a key component of precision oncology, are of particular importance because there are multiple lines of treatment that can be probed for effectiveness. A variety of response parameters, perhaps too many, have been introduced; these include PERCIST (30), Deauville (31), PECRIT (combined RECIST 1.1 and PERCIST), and PERCIMT PET (Response Evaluation Criteria for Immunotherapy) (32,33). Under baseline conditions, ${ }^{18} \mathrm{~F}-\mathrm{FDG}$ tumor uptake provides information about tumor glycolytic activity. Early changes in glucose metabolic activity in response to therapy are a powerful indicator of a favorable treatment response (34). In fact, a pharmacologic treatment cannot and will not be effective if it is not associated with a precipitous reduction in tumor ${ }^{18} \mathrm{~F}-\mathrm{FDG}$ uptake. This paradigm became evident in preclinical and clinical studies. Mai 
TABLE 1

Imaging Biomarkers

\begin{tabular}{|c|c|}
\hline Biomarker class & $\begin{array}{l}\text { Information provided } \\
\text { (question answered) }\end{array}$ \\
\hline Diagnostic & Is the disease present? \\
\hline Phenotypic & What is the biologic behavior? \\
\hline Prognostic & What is the outcome? \\
\hline Predictive & Is the therapeutic target expressed? \\
\hline $\begin{array}{l}\text { Intermediate } \\
\text { endpoint }\end{array}$ & $\begin{array}{l}\text { Does the disease respond } \\
\text { to treatment? }\end{array}$ \\
\hline Pharmacokinetic & What is the drug distribution? \\
\hline Pharmacodynamic & Is the therapeutic target inhibited? \\
\hline
\end{tabular}

et al. (35) and Clark et al. (36) demonstrated that early and precipitous reductions in ${ }^{18} \mathrm{~F}$-FDG uptake predicted the responses of orthotopic glioblastomas to epidermal growth factor receptor inhibition. Numerous clinical studies confirmed this pattern (37-39). This evidence explains the continuing growth and broad adoption of clinical PET/CT imaging.

\section{Established Therapies}

Adjuvant ${ }^{131}$ I therapy for thyroid cancer, the first example of successful nuclear theranostics, continues to be a key application of nuclear medicine (40). Bone pain palliation with ${ }^{89} \mathrm{Sr}$ and ${ }^{153} \mathrm{Sm}$ was introduced decades ago (41). Both have largely been replaced by ${ }^{223} \mathrm{Ra}$ treatment, which results in symptomatic relief and prolongs the survival of prostate cancer patients (42). In collaboration with interventional radiology, ${ }^{90} \mathrm{Y}$ therapy for metastatic disease to the liver is an important component of late-stage therapy (43). CD20-targeted therapies for lymphoma were conceptually intriguing and efficacious, but given the competition with similarly effective nonradioactive approaches, these therapies did not gain widespread clinical acceptance for the treatment of lymphoma (44).
So far we have highlighted the strength of nuclear medicine that arises from established imaging biomarkers and therapies. However, new opportunities in the diagnosis of and therapy for NETs, paraganglioma, pheochromocytoma, and bone metastatic disease-as well as, predictably in the near future, prostate cancer-are about to reshape nuclear medicine. Appropriate training will be critically important for integrating these approaches into the overall management of cancer patients.

\section{OPPORTUNITIES}

\section{New Imaging Biomarkers}

The FDA approved several imaging probes in recent years. These include the synthetic amino acid ${ }^{18} \mathrm{~F}$-fluciclovine (45) and the amino-alcohol ${ }^{11} \mathrm{C}$-choline for use in prostate cancer (46) as well as ${ }^{68} \mathrm{Ga}$-DOTATATE (47) and, in the near future, ${ }^{68} \mathrm{Ga}$-DOTATOC (48) to exploit the high level of somatostatin receptor expression of NETs. Several radiotracers targeting amyloid (49) and tau (50) are undergoing clinical evaluations and represent a key element in dementia research.

Important imaging probes that will become available include various ${ }^{68} \mathrm{Ga}$ - and ${ }^{18} \mathrm{~F}$-labeled PSMA ligands (51-55). Additional imaging probes that should become available-although phase 3 clinical imaging trials without commercial sponsors will be difficult to complete-include L-amino-acid transporter-1 (LAT-1) substrates, such as ${ }^{11} \mathrm{C}$-methionine $(56),{ }^{18} \mathrm{~F}$-tyrosine $(57)$, and ${ }^{18} \mathrm{~F}$-FDOPA $(58,59)$, for use in brain tumors, movement disorders, and NETs. The need to apply common sense to the approval processes in the absence of potent industry sponsorship has been discussed elsewhere (60).

PET imaging can interrogate the whole body for the expression of therapeutic targets (61). The presence and degree of target expression are associated with a therapy response. Thus, PET imaging probes have been introduced as predictive biomarkers. For instance, the degree of somatostatin receptor expression in patients with NETs, as measured with ${ }^{68} \mathrm{Ga}$-DOTATATE/TOC/NOC, permits predictions of peptide receptor radionuclide therapy responses (62). PSMA expression determined with PET stratifies patients toward

TABLE 2

Selected FDA-Approved Imaging Biomarkers

\begin{tabular}{|c|c|c|c|c|}
\hline Probe & Target & Process & Application & Biomarker type \\
\hline${ }^{18} \mathrm{~F}-\mathrm{FDG}$ & $\begin{array}{l}\text { Hexokinase 2, GLUT1, } \\
\text { GLUT3, GLUT4 }\end{array}$ & Glucose metabolism & $\begin{array}{l}\text { Cancer, cardiovascular disease, } \\
\text { neurodegenerative diseases, } \\
\text { infectious disease }\end{array}$ & $\begin{array}{l}\text { Diagnostic, phenotypic, } \\
\text { prognostic, intermediate } \\
\text { endpoint }\end{array}$ \\
\hline${ }^{18} \mathrm{~F}-\mathrm{NaF}$ & Hydroxyapatite & Bone remodeling & $\begin{array}{l}\text { Primary and metastatic } \\
\text { bone cancers, degenerative/ } \\
\text { inflammatory bone disease }\end{array}$ & Diagnostic, prognostic \\
\hline${ }^{11} \mathrm{C}$-choline & Choline kinase & Lipid metabolism & (Prostate) cancer & Diagnostic, phenotypic \\
\hline${ }^{68} \mathrm{Ga}-\mathrm{DOTATATE}$ & Somatostatin receptors & $\begin{array}{l}\text { Somatostatin receptor } \\
\text { density }\end{array}$ & NETs & Diagnostic, predictive \\
\hline${ }^{18} \mathrm{~F}$-fluciclovine & LAT1 & Amino acid transport & (Prostate) cancer & Diagnostic, phenotypic \\
\hline${ }^{82} \mathrm{Rb}$ & $\mathrm{Na}+/ \mathrm{K}+$ ion exchange & Myocardial blood flow & Detection of CAD & Diagnostic \\
\hline${ }^{13} \mathrm{~N}$-ammonia & $\begin{array}{l}\text { Incorporation into glutamine } \\
\text { by glutamine synthetase }\end{array}$ & Myocardial blood flow & Detection of CAD & Diagnostic \\
\hline${ }^{123}$-ioflupane & $\begin{array}{l}\text { Presynaptic dopamine } \\
\text { transporter }\end{array}$ & Dopamine transport & Movement disorders & Diagnostic \\
\hline $\begin{array}{l}{ }^{18} \text { F-labeled florbetapir, } \\
\text { florbetaben, and } \\
\text { flutemetamol }\end{array}$ & Amyloid & Amyloid deposition & $\begin{array}{l}\text { Progressive } \\
\text { neurodegenerative diseases }\end{array}$ & Diagnostic, prognostic \\
\hline
\end{tabular}


PSMA-targeted molecular radiotherapy (63). Androgen receptor imaging provides prognostic information in patients with castrationresistant metastatic prostate cancer (64). The effects of antiandrogen drugs can be directly visualized using PET imaging of androgen receptor expression (65) (pharmacodynamic biomarkers).

Thus, PET biomarkers are now widely available for clinical and research uses. Nevertheless, they are still vastly underused, accounting for the small number of PET scans performed annually in the United States compared with the use of CT in oncology $(66,67)$.

However, treating and providing physicians as well as regulatory agencies and, at times, even health insurance companies increasingly recognize the value of imaging biomarkers. Deploying the large portfolio of PET probes and biomarkers rationally and effectively requires knowledge of biology, metabolism (e.g., of cancer cells, the brain, benign infectious diseases, and normal organs), drugtarget interactions, cell surface receptor expression and its association with the treatment response, and a solid background in medicine and associated clinical disciplines. A detailed description of the many radionuclide imaging biomarkers and approaches that are available for neurologic and cardiac applications is beyond the scope of this review. The growing number of imaging biomarkers strongly suggests the continuing growth of nuclear medicine and molecular imaging. However, also evident is the fact that mastering the increasing diversity of diagnostic nuclear medicine and molecular imaging requires much more than 4 mo of training.

\section{Precision Therapeutics}

Precision or personalized medicine is often described nearly exclusively in the context of genomics. Underlying this concept is the notion that actionable cancer cell mutations may represent a cancer's Achilles heel. Such actionable mutations include those of the epidermal growth factor receptor (68), BCR-ABL (69), BRAF (70), and many others. It was the success of imatinib for the treatment of chronic myelogenous leukemia (69) and gastrointestinal stromal tumors that raised the hope that single oncogenic drivers could be identified and targeted successfully for most cancers.

This topic was investigated in the NIC-MATCH trial (The Molecular Analysis for Therapy Choice trial) (71). On the basis of specific mutations, patients received FDA-approved targeted treatments to determine whether these mutation-specific therapies were effective, regardless of cancer type. Genotyping was completed in more than $80 \%$ of more than 700 patients. However, fewer than $10 \%$ of patients harbored an actionable mutation, and only $2.5 \%$ of patients eventually received matching treatment (72). Another provocative result was that targeted therapy based on tumor molecular profiling did not improve patient outcomes compared with conventional, agnostic, nontargeted therapy (73). In that randomized trial (73), patients with any solid tumor accessible to biopsy were included, and large-scale genomic testing was performed. Only patients with alterations in 1 of 3 molecular pathways (hormone receptor, $\mathrm{PI} 3 \mathrm{~K} / \mathrm{AKT} / \mathrm{mTOR}$, and $\mathrm{RAF} / \mathrm{MEK}$ ) received 11 available matching molecularly targeted agents (erlotinib, lapatinib plus trastuzumab, sorafenib, imatinib, dasatinib, vemurafenib, everolimus, abiraterone, letrozole, and tamoxifen). Seven hundred fourteen patients were enrolled, and $40 \%$ had an actionable molecular alteration. These patients were randomized to receive the matched targeted therapy or the physician's choice, essentially, empiric, nontargeted cytotoxic treatments. There was no difference in progression-free survival, the primary endpoint, 12 mo after the start of therapy.
Letai (72) proposed an approach that detects not just "somatic cancer mutations but rather somatic cancer vulnerabilities ...." To achieve this goal, he suggested pursuing "functional precision medicine in cancer," including "omics," clinical experience, and "functional assays." One unique functional assay on the wholebody level is PET imaging.

In summary, the goal of precision oncology has thus far remained largely elusive. Nuclear medicine techniques and assays usually are not discussed in the context of precision medicine, defined as the right treatment (drug or others), for the right patient, at the right dose, at the right time. Nevertheless, no discipline other than nuclear theranostics can provide noninvasive readouts of target expression and address the target structure successfully.

\section{Nuclear Theranostics}

Several powerful nuclear theranostic approaches are already being clinically used or are undergoing phase 3 clinical trial evaluations. The NETTER trial is an extremely convincing example of precision oncology (14). The expression of somatostatin receptors as a predictive biomarker was determined with ${ }^{111} \mathrm{In}$-DOTATATE imaging. Only patients with sufficient somatostatin receptor expression qualified for treatment with ${ }^{177} \mathrm{Lu}$-DOTATATE. Whole-body imaging, now done with ${ }^{68} \mathrm{Ga}$-DOTATATE, has a significant advantage over invasive tissue sampling, which is limited to few disease sites and is subject to tumor heterogeneity and sampling errors. Somatostatin receptortargeted PET molecular imaging ( ${ }^{68} \mathrm{Ga}$-DOTATATE; NETSPOT [Advanced Accelerator Applications]) and molecular radiotherapy ( ${ }^{177}$ Lu-DOTATATE; Lutathera [Advanced Accelerator Applications]) are now approved by the FDA and are reimbursed by the Centers for Medicare \& Medicaid Services. The NETTER trial results unequivocally demonstrated highly significant benefits in progression-free and overall survival of patients undergoing ${ }^{177} \mathrm{Lu}-$ DOTATATE versus standard-of-care treatment. This outcome was met with great business interest, and Novartis acquired Advanced Accelerator Applications, the licensee of Lutathera, for \$3.9 billion in the fall of 2017. Since then, Novartis has expanded its nuclear theranostic portfolio to include PSMA-targeted diagnostics and therapeutics.

On the basis of a phase 2 multicenter trial, ${ }^{131}$ I-iobenguane (Azedra; Progenics Pharmaceuticals), a guanethidine derivative that is a substrate for norepinephrine reuptake transporters, was approved by the FDA for the therapy of pheochromocytoma and paraganglioma (15). In brief, in that study of 68 patients, high-specific-activity ${ }^{131}$ I-metaiodobenzylguanidine induced durable reductions in antihypertensive medication use, the primary endpoint of the study. Ninety-two percent of patients had stable disease or a partial response within 12 mo of treatment. Overall survival was $36.7 \%$. The treatment was not without toxicity, which included nausea, myelosuppression, and fatigue. Given the small number of patients with these orphan diseases, randomized trials are not feasible and in fact would be detrimental to patients' best interests. Appropriately, on the basis of a lack of alternative therapies, effectiveness data, and side effect profile, the product received FDA approval.

Public awareness and attention have recently focused on PSMAtargeted theranostics. Many diagnostic and several therapeutic compounds have been introduced. Various radiation approaches, including $\beta$-particle radiation with ${ }^{177} \mathrm{Lu}(63)$ and ${ }^{131} \mathrm{I}$ (74) and $\alpha$-particle radiation with ${ }^{225} \mathrm{Ac}(75)$, underwent more or less rigorous trials (Fig. 2). Both diagnostics and therapeutics are already in routine clinical use worldwide. The presence, extent, and degree of PSMA expression are determined with ${ }^{68} \mathrm{Ga}$-PSMA, and patients are 


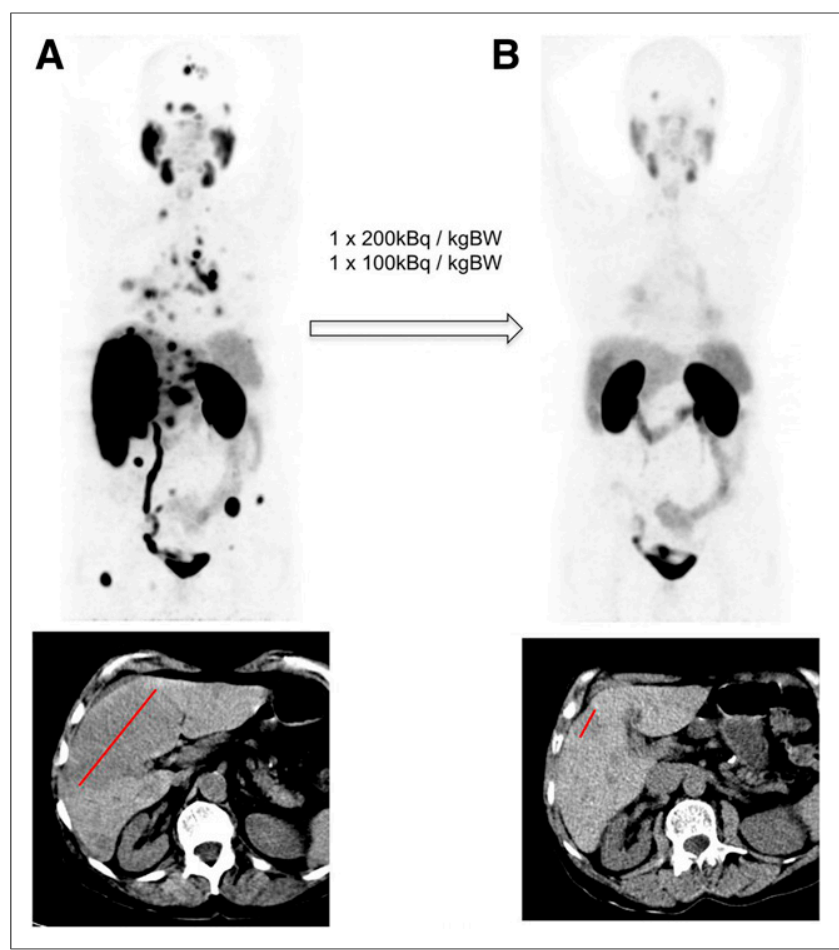

FIGURE 2. Baseline (A) and restaging (B) of ${ }^{68} \mathrm{Ga}-\mathrm{PSMA}-11 \mathrm{PET}$ (maximum-intensity projection) (top) and CT (bottom) in patient with histologically proven prostate cancer lung and liver metastases. Follow-up scan (B) was done after ${ }^{225} \mathrm{Ac}-\mathrm{PSMA}-617$ treatment $(200 \mathrm{kBq} / \mathrm{kg})$. BW = body weight. (Reprinted with permission from (75).)

stratified for treatment with ${ }^{177} \mathrm{Lu}$-PSMA. In fact, in 1 prospective phase 2 study, glucose metabolic phenotyping together with PSMA receptor expression assessments was used to qualify patients for ${ }^{177} \mathrm{Lu}$-PSMA-617 therapy (63). High prostate-specific antigen response rates suggested that further refinements in patient stratification are possible. That study (63) exploited PET metabolic phenotyping to enrich the population of patients who can benefit from molecular radiotherapy targeting of PSMA further. An ongoing phase 3 clinical trial (VISION) will provide definitive evidence for or against the effectiveness of this nuclear theranostic approach in late-stage prostate cancer patients.
In the United States, large-scale phase 3 trials testing the diagnostic performance of various PSMA ligands labeled with ${ }^{18} \mathrm{~F}$ or ${ }^{68} \mathrm{Ga}$ are approaching completion or have been published $(76,77)$. It appears quite likely that diagnostic compounds will gain FDA approval before PSMA-targeted therapeutic compounds are reviewed and evaluated if the phase 3 clinical trials provide the anticipated results.

Other equally exciting nuclear theranostic approaches are emerging. The Heidelberg group has identified the fibroblast activation protein as a tumor stroma target (78) and has developed a series of quinolone-based fibroblast activation protein ligands (79). ${ }^{68} \mathrm{Ga}-$ labaled ligands accumulate with high specificity in target structures. Importantly, extensive stroma components have been observed across many cancers $(80,81)$, suggesting that a theranostic radionuclide pair for targeting various cancers could emerge.

Table 3 shows a list of widely adopted and emerging theranostic radionuclide pairs.

\section{${ }^{68} \mathrm{Ga}$-DOTATATE and ${ }^{177} \mathrm{Lu}$-DOTATATE}

Fifteen thousand patients will be diagnosed with NETs in the United States in 2019. Because of long survival durations, the prevalence of the disease is higher, at about 35,000 . Before ${ }^{68} \mathrm{Ga}-$ DOTATATE availability, 20,000 ${ }^{111}$ In-DOTATATE scans were performed annually in the United States. Reasonable insurance companies should replace all of these scans with ${ }^{68} \mathrm{Ga}$-DOTATATE PET/CT (NETSPOT). Half of the 15,000 NET patients will undergo surgery. The remaining approximately 7,500 patients are nonsurgical candidates who might benefit from ${ }^{177} \mathrm{Lu}$-DOTATATE (Lutathera) treatment. Because each patient would undergo $4{ }^{177}$ Lu-DOTATATE cycles, a total of 30,000 cycles would be needed annually in the United States (Table 4).

\section{${ }^{68} \mathrm{Ga}-\mathrm{PSMA}$ and ${ }^{177} \mathrm{Lu}-\mathrm{PSMA}$}

Although not yet FDA approved, PSMA-targeted radioligand therapy (RLT) is without any doubt entering the standard armamentarium against advanced prostate cancer. A total of 180,000 new cases occur annually in the United States (82). Sixty thousand of these patients are diagnosed with high-risk disease (83). Approximately 60,000 of the 120,000 patients with low- or intermediate-risk disease experience biochemical recurrence. All of the latter and all 60,000 patients with high-risk disease are candidates for ${ }^{68} \mathrm{Ga}$ - or ${ }^{18} \mathrm{~F}$-PSMA imaging for appropriate staging and disease management, including radiation planning (Fig. 3) (84).

TABLE 3

Current and Future Nuclear Theranostic Approaches

\begin{tabular}{|c|c|c|}
\hline Nuclear theranostic pair & Target & Application \\
\hline${ }^{68} \mathrm{Ga}-$ and ${ }^{177} \mathrm{Lu}$-DOTATATE & Somatostatin receptors & NETs \\
\hline${ }^{68} \mathrm{Ga}-$ and ${ }^{177} \mathrm{Lu}-\mathrm{PSMA}$ & PSMA & Prostate cancer \\
\hline${ }^{123} \mathrm{I}$ - and ${ }^{131} \mathrm{I}$-iobenguane & Norepinephrine reuptake transporter & $\begin{array}{l}\text { Neuroblastoma, paraganglioma, } \\
\text { pheochromocytoma }\end{array}$ \\
\hline${ }^{68} \mathrm{Ga}-$ and ${ }^{177} \mathrm{Lu}-\mathrm{FAPI}$ & Fibroblast activation protein & Multiple cancers \\
\hline${ }^{68} \mathrm{Ga}-$ and ${ }^{177} \mathrm{Lu}-3 \mathrm{BP}-227$ NTR1 antagonist & Neurotensin receptor 1 & Pancreatic cancer \\
\hline${ }^{124} \mid$ - and ${ }^{131} \mid$-girentuximab & Carbonic anhydrase 9 & Renal cell cancer \\
\hline $123 \mid-$ and ${ }^{131} \mid$-iodine & Sodium/iodide symporter & Thyroid diseases \\
\hline
\end{tabular}


TABLE 4

Projected Annual Numbers of Nuclear Theranostic Interventions

\begin{tabular}{|c|c|c|c|c|c|}
\hline & Theranostic & No. of patients/y & No. of cycles & Reimbursement (U.S. \$) & Revenue (U.S. \$/y) \\
\hline \multirow[t]{3}{*}{ Diagnostic imaging } & ${ }^{68} \mathrm{Ga}-\mathrm{DOTATATE}$ & 20,000 & & 3,000 & 60 (Mil U.S. \$/y) \\
\hline & ${ }^{68} \mathrm{Ga}-\mathrm{PSMA}$ & 160,000 & & 3,000 & 480 (Mil U.S. \$/y) \\
\hline & Total diagnostic & 198,000 & & & 540 (Mil U.S. \$/y) \\
\hline \multirow[t]{3}{*}{ Therapeutic } & ${ }^{177}$ Lu-DOTATATE & 7,500 & 30,000 (4 cycles/patient) & $40,000 /$ cycle & 1.2 (Bil U.S. \$/y) \\
\hline & ${ }^{177}$ Lu-PSMA & 40,000 & 160,000 (4 cycles/patient) & $20,000 /$ cycle $^{*}$ & 3.2 (Bil U.S. \$/y) \\
\hline & Total therapeutic & 47,500 & 190,000 & & 4.4 (Bil U.S. \$/y) \\
\hline $\begin{array}{l}\text { Total nuclear } \\
\text { theranostics }\end{array}$ & & & & & 4.940 (Bil U.S. \$/y) \\
\hline
\end{tabular}

${ }^{\star}$ Assumed charge/cycle.

About 40,000 prostate cancer patients who die each year in the United States (82) are potential candidates for RLT. PSMA imaging would be required in all of them for appropriate RLT stratification. Thus, we estimate that the total number of annual PSMA PET/CT studies in the United States would amount to 160,000 .

Forty-thousand end-stage prostate cancer patients treated with RLT would create the need for 160,000-240,000 treatment cycles if $4-6$ cycles were administered per patient. If RLT were to be given to hormone-sensitive patients, then a much larger number of treatment cycles would be required (Table 4).

The financial implications are striking, as shown in Table 4. Assuming current NETSPOT reimbursement levels, diagnostic studies targeting somatostatin receptors and PSMA could result in a revenue potential of close to $\$ 500$ million. Adding 30,000 treatment cycles for 7,500 NET patients and 160,000 cycles for 40,000 prostate cancer patients would yield close to 190,000 treatment cycles annually. Hypothetical per-cycle technical reimbursement values are provided in Table 4 (professional fees are currently close to $\$ 0$ ).

Total annual revenue for nuclear theranostics would then be estimated at approximately $\$ 5$ billion. The 3 underlying assumptions for Table 4 are as follows: PSMA-targeted theranostics will meet phase 3 endpoints and will be FDA approved, reimbursement levels will be close to enumerations for standard oncology biologic agents, and therapies will be restricted to late-stage disease.

However, even these conservative numbers raise logistical questions, such as, "How many cycles can be done at 1 site?" Once such an approach is implemented, patients would spend no more than 1-2 $\mathrm{h}$ in outpatient clinics. With appropriate staffing (e.g., nurses, technologists), 8-10 cycles/d could be administered in high-volume centers. Administering 190,000 cycles annually (160,000 for prostate cancer and 30,000 for NETs) with the assumption of 261 working days in the United States would require the administration of 728 cycles/d. In other words, 70 treatment centers administering about 10 cycles/d would be required to meet this demand in the United States. The potential for great demand raises concern about lack of human resources. However, demand like that described here will not materialize for several years. If nuclear medicine will not adapt to increasing demand, then other specialties will. However, models in Europe, Australia, and Asia clearly have shown that a welltrained nuclear medicine work force can lead the field of theranostics with great success.

\section{CHALLENGES}

Financial Viability of Nuclear Medicine, Molecular Imaging, and Nuclear Theranostics

Interpretations of PET/CT studies can require as long as $1 \mathrm{~h}$ if the process includes obtaining patient history, which is often complex, and conducting careful comparisons with prior anatomic and molecular imaging studies. Given these complexities, professional Centers for Medicare \& Medicaid Services reimbursement rates for ${ }^{18}$ F-FDG PET imaging studies $(\sim 100)$ are low (Table 5). Although artificial intelligence and deep learning may aid in and expedite image interpretation and reporting in the future, these fields are still immature.

Reimbursement rates for other nuclear medicine imaging studies are even lower (Table 5). There is an obvious imbalance between high technical (operational) and low professional (image interpretation/ reporting) reimbursements in the United States. Unlike radiology, nuclear medicine is not a high-volume operation. It is therefore difficult to maintain a financially viable practice on the basis of professional reimbursement fees. Stand-alone nuclear medicine clinics do not exist in any significant numbers in the United States. In U.S. academic institutions and community hospitals, almost all nuclear medicine programs are divisions of radiology departments. These are, in our view, constructs of administrative and fiscal convenience

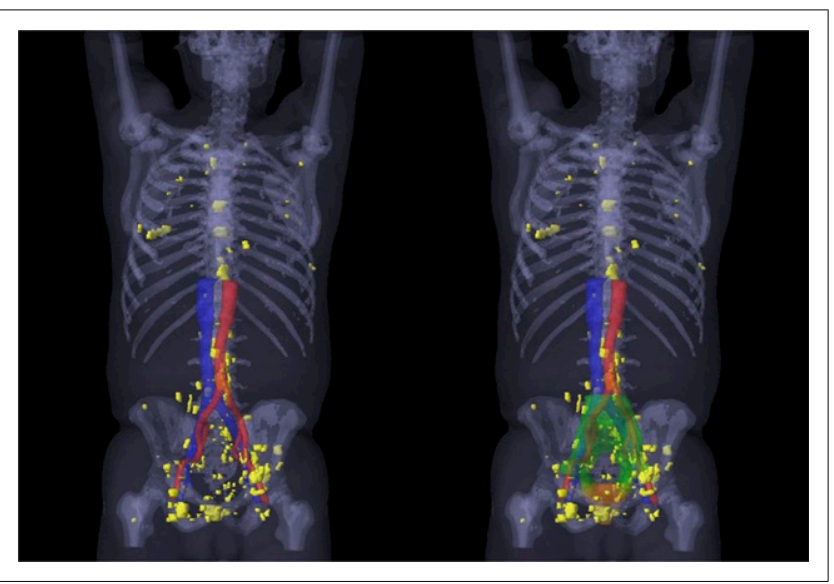

FIGURE 3. 3-Dimensional map of ${ }^{68} \mathrm{Ga}-\mathrm{PSMA}-11-$ positive lesions (yellow) in all 52 patients with recurrence outside standard radiation target volumes. Findings suggested that ${ }^{68} \mathrm{Ga}$-PSMA imaging changes radiation target volumes. (Reprinted with permission from (84).) 
TABLE 5

Examples of Professional and Technical Reimbursement Rates (Medicare) for Nuclear Medicine Studies and Therapies

\begin{tabular}{|c|c|c|c|}
\hline Study or therapy & Technical fee $(\$)$ & Professional fee $(\$)$ & Radiopharmaceutical fee $(\$)^{\star}$ \\
\hline Whole-body PET/CT & $1,375.61$ & 135.51 & \\
\hline Cardiac PET (viability) & $1,229.29$ & 72.44 & \\
\hline Neuro-PET & 1375.61 & 73.53 & \\
\hline Thyroid cancer therapy & 50.82 & 90.10 & \\
\hline \multicolumn{4}{|l|}{ Thyroid scan } \\
\hline 131| whole body & 58.38 & 28.47 & \\
\hline 131I whole body + uptake & 283.27 & 42.17 & \\
\hline Bone scan & 270.65 & 43.25 & \\
\hline Cardiac SPECT & 283.27 & 68.83 & \\
\hline Renal scan (mercaptoacetyltriglycine) & 312.10 & 69.92 & \\
\hline${ }^{177} \mathrm{Lu}$-lutetium DOTATATE (Lutathera) treatment & 50.45 & 100.19 & 26,456 \\
\hline${ }^{223}$ Ra-radium dichloride (Xofigo; Bayer) & 100.19 & 50.45 & 14,000 \\
\hline
\end{tabular}

${ }^{*}$ Radiotracer fees are given only for therapeutic studies.

and often do not favor or foster innovation in molecular imaging and therapy. Nuclear medicine clinics are important independent departments in major academic health care systems worldwide.

The lack of economic pressures in not-for-profit health care systems accounts, in part, for thriving nuclear medicine departments. In other words, the fiscal viability of nuclear medicine is less relevant in the European or Australian systems (mostly not-for-profit) than it is in the United States (for-profit) health care model. In public health care systems, the net profit is less relevant than the net benefit for patients. The independence of clinical departments is thus a matter of medical impact rather than financial profitability.

However, nuclear theranostics not only provides great opportunities for patients and thus for the physicians taking care of these patients but also has the potential to change the nuclear medicine business model.

\section{Training and Licensing}

Our aim here is to stimulate discussions about the best way forward for nuclear medicine, molecular imaging, and nuclear theranostic training as well as certification requirements.

Considering the increasingly broad scope of nuclear medicine, molecular imaging, and molecular radiotherapy, the need for much improved training and education is evident. Table 6 delineates the multitude of pathways for nuclear medicine training in the United States. Standardization is needed. Nuclear Regulatory Commissionauthorized user accreditation for radiologists and radiation oncologists after only 4 mo of training defies logic.

The molecular characterization of diseases is becoming increasingly complex, requiring a special and different skill set. PET/CT or SPECT/CT studies can be interpreted jointly with radiology (85).

TABLE 6

Current Training Pathways for Nuclear Medicine in United States

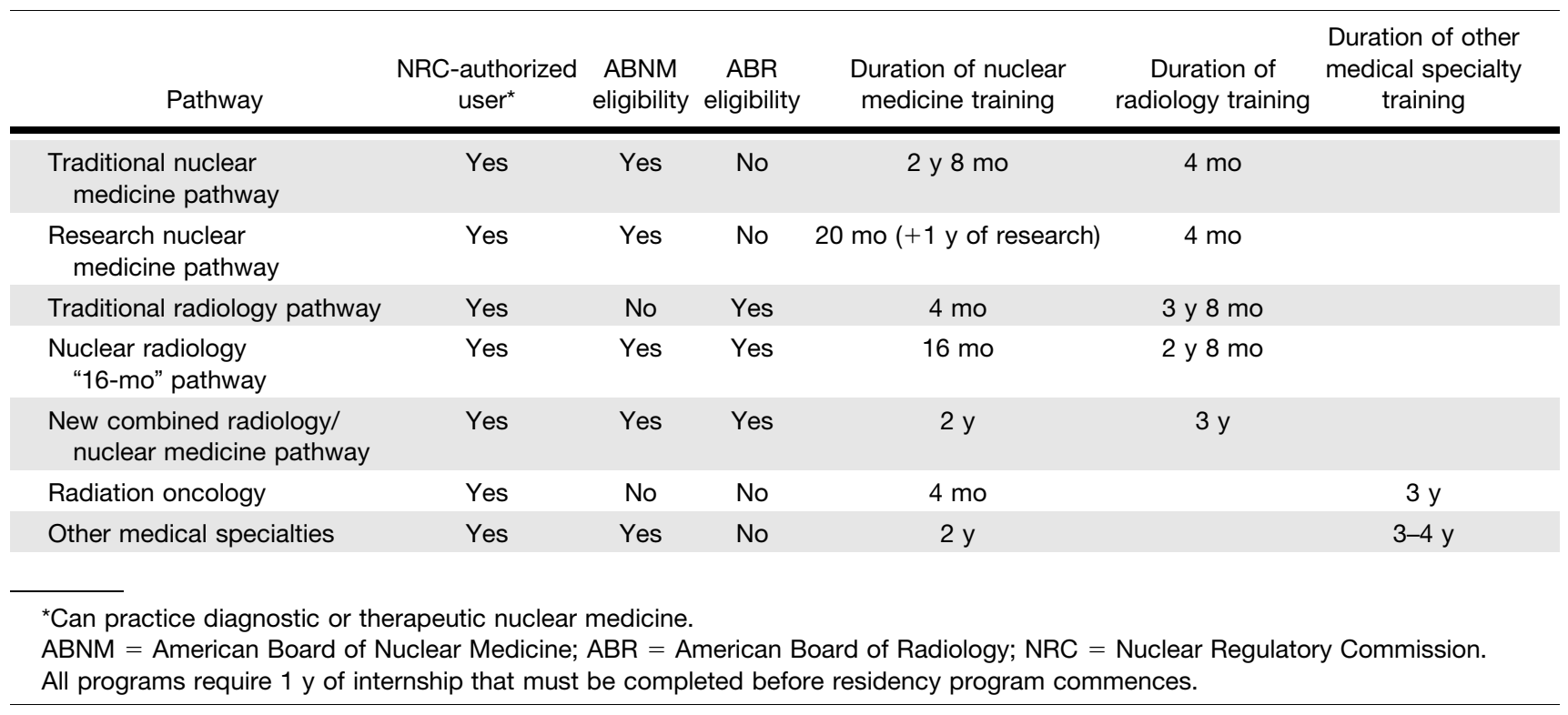




\begin{tabular}{|c|c|c|}
\hline Year of training & Curriculum & Requirements \\
\hline \multirow[t]{2}{*}{ First year } & $\begin{array}{l}\text { Basic science and conventional nuclear } \\
\text { medicine (planar imaging and SPECT) }\end{array}$ & $\begin{array}{l}\text { Radiation physics, radiochemistry, radiation biology, } \\
\text { and instrumentation }\end{array}$ \\
\hline & Introduction to cross-sectional imaging & $\begin{array}{l}\text { Conventional nuclear medicine studies for musculoskeletal system, } \\
\text { myocardial perfusion, endocrinology, gastrointestinal tract, } \\
\text { inflammation, infection, pulmonary function, neurology, } \\
\text { oncology, and urinary tract }\end{array}$ \\
\hline \multirow[t]{2}{*}{ Second year } & CT and cross-sectional imaging & PET (PET/CT and PET/MRI), CT, MRI \\
\hline & & SPECT/CT \\
\hline \multirow[t]{3}{*}{ Third year } & $\begin{array}{l}\text { Molecular radiotherapy and } \\
\text { nuclear theranostics }\end{array}$ & Dosimetry \\
\hline & & Therapy-related radiobiology \\
\hline & & Patient management \\
\hline \multirow[t]{2}{*}{ Fourth year } & $\begin{array}{l}\text { Advanced diagnostic and therapeutic } \\
\text { nuclear medicine }\end{array}$ & Radiology (elective) \\
\hline & & $\begin{array}{l}\text { Selected focus domains: cardiac imaging, neuroimaging, } \\
\text { oncology, nuclear theranostics }\end{array}$ \\
\hline
\end{tabular}

However, interpreting these studies jointly does not rule out the independence - in content and administratively — of the 2 specialties, which have substantial overlap but are inherently different. Nuclear medicine has deep roots in biology, biochemistry, metabolism, and medicine. Radiology is largely anchored in anatomy. Molecular radiotherapy and nuclear theranostics necessitate a thorough understanding of disease entities and molecular targets, sequence of therapies, radiation biology, radiation physics, instrumentation, internal dosimetry, management of oncologic patients and treatment side effects, and radiation safety, among other aspects.

The emergence of new radionuclide therapies has created an urgent need to reevaluate nuclear medicine training programs in the United States. The International Atomic Energy Agency recommends 4 y of dedicated nuclear medicine training (after 1-2 y of internship) (86). This model has been applied in various places in Europe and Australia, where 4- to 5-y programs have been highly successful (87).

In Table 7, we propose a 4-y training program (not reflecting official American Board of Nuclear Medicine or Society of Nuclear Medicine and Molecular Imaging opinions) that matches the educational standards applied worldwide. Board certification and licensing should be contingent on meeting these standards and requirements. The first $3 \mathrm{y}$ of the training program (Tab. 7) should be mandatory for any physician seeking dual board certification in nuclear medicine (e.g., radiologists, radiation oncologists, cardiologists, or internists). Whatever training model and certification process are adopted, the nuclear medicine community needs to approach these discussions with confidence, competence, and optimism. The diagnostic and therapeutic services developed in nuclear medicine will persist, but without adaptation and change, they will be delivered by others.

\section{CONCLUSION}

The future of nuclear medicine is bright, but significant challenges exist. Nuclear medicine represents a vibrant and viable specialty encompassing molecular imaging and molecular radiotherapy. Nuclear theranostics is the most convincing example of precision medicine, as whole-body target expression that provides information for optimizing treatment approaches can be measured noninvasively. Completely revised board certification and licensing standards are needed to achieve optimized and integrated patient care.

\section{DISCLOSURE}

Johannes Czernin, MD, editor-in-chief of The Journal of $\mathrm{Nu}$ clear Medicine, has indicated that he is a founder and board member of Sofie Biosciences; he holds equity in the company and in intellectual property invented by him, patented by the University of California, and licensed to Sofie Biosciences. He has also indicated that he is a founder and board member of Trethera Therapeutics; he holds equity in the company and in intellectual property invented by him, patented by the University of California, and licensed to Triangle. No other potential conflict of interest relevant to this article was reported.

\section{REFERENCES}

1. Accreditation Council for Graduate Medical Education (ACGME). https://apps. acgme.org/ads/Public/Programs/Search. Accessed June 21, 2019.

2. Segall GM, Grady EE, Fair JR, Ghesani MV, Gordon L. Nuclear medicine training in the United States. J Nucl Med. 2017;58:1733-1734.

3. ACGME. (Public Reports 2017). https://apps.acgme.org/ads/Public/Reports/ReportRun? ReportId $=3 \&$ Current Year $=2017 \&$ Academic YearId $=2017$.

4. Hillner BE, Siegel BA, Liu D, et al. Impact of positron emission tomography/ computed tomography and positron emission tomography (PET) alone on expected management of patients with cancer: initial results from the National Oncologic PET Registry. J Clin Oncol. 2008;26:2155-2161.

5. Hillner BE, Siegel BA, Hanna L, et al. Impact of ${ }^{18} \mathrm{~F}-\mathrm{FDG}$ PET used after initial treatment of cancer: comparison of the National Oncologic PET Registry 2006 and 2009 cohorts. J Nucl Med. 2012;53:831-837.

6. Beyer T, Townsend DW, Brun T, et al. A combined PET/CT scanner for clinical oncology. J Nucl Med. 2000;41:1369-1379.

7. Silverman DHS, Small GW, Chang CY, et al. Positron emission tomography in evaluation of dementia: regional brain metabolism and long-term outcome. JAMA. 2001;286:2120-2127. 
8. Schelbert HR. ${ }^{18}$ F-deoxyglucose and the assessment of myocardial viability. Semin Nucl Med. 2002;32:60-69.

9. Czernin J, Phelps ME. Positron emission tomography scanning: current and future applications. Annu Rev Med. 2002;53:89-112.

10. Coleman RE, Hillner BE, Shields AF, et al. PET and PET/CT reports: observations from the National Oncologic PET Registry. J Nucl Med. 2010;51:158-163.

11. Berman DS, Hachamovitch R, Shaw LJ, et al. Roles of nuclear cardiology, cardiac computed tomography, and cardiac magnetic resonance: noninvasive risk stratification and a conceptual framework for the selection of noninvasive imaging tests in patients with known or suspected coronary artery disease. J Nucl Med. 2006;47:1107-1118.

12. Delbeke D, Segall GM. Status of and trends in nuclear medicine in the United States. J Nucl Med. 2011;52(suppl):24S-28S.

13. van Essen M, Sundin A, Krenning EP, Kwekkeboom DJ. Neuroendocrine tumours: the role of imaging for diagnosis and therapy. Nat Rev Endocrinol. 2014;10:102.

14. Strosberg J, El-Haddad G, Wolin E, et al. Phase 3 trial of ${ }^{177} \mathrm{Lu}$-Dotatate for midgut neuroendocrine tumors. N Engl J Med. 2017;376:125-135.

15. Pryma DA, Chin BB, Noto RB, et al. Efficacy and safety of high-specific-activity ${ }^{131}$ I-MIBG therapy in patients with advanced pheochromocytoma or paraganglioma. J Nucl Med. 2019;60:623-630.

16. Eiber M, Fendler WP, Rowe SP, et al. Prostate-specific membrane antigen ligands for imaging and therapy. J Nucl Med. 2017;58(suppl):67S-76S.

17. Valliant JF. A bridge not too far: linking disciplines through molecular imaging probes. J Nucl Med. 2010;51:1258-1268.

18. Krasniqi A, D'Huyvetter M, Devoogdt N, et al. Same-day imaging using small proteins: clinical experience and translational prospects in oncology. J Nucl Med. 2018;59:885-891.

19. Waaijer SJH, Kok IC, Eisses B, et al. Molecular imaging in cancer drug development. J Nucl Med. 2018;59:726-732.

20. Donin NM, Reiter RE. Why targeting PSMA is a game changer in the management of prostate cancer. $J$ Nucl Med. 2018;59:177-182.

21. Moek KL, Giesen D, Kok IC, et al. Theranostics using antibodies and antibodyrelated therapeutics. $J$ Nucl Med. 2017;58(suppl):83S-90S.

22. Barthel H, Sabri O. Clinical use and utility of amyloid imaging. J Nucl Med. 2017;58:1711-1717.

23. Iskandrian AE, Hage FG, Shaw LJ, Mahmarian JJ, Berman DS. Serial myocardial perfusion imaging: defining a significant change and targeting management decisions. JACC Cardiovasc Imaging. 2014;7:79-96.

24. Salmanoglu E, Kim S, Thakur ML. Currently available radiopharmaceuticals for imaging infection and the holy grail. Semin Nucl Med. 2018;48:86-99.

25. Buck AK, Herrmann K, Stargardt T, Dechow T, Krause BJ, Schreyogg J. Economic evaluation of PET and PET/CT in oncology: evidence and methodologic approaches. J Nucl Med. 2010;51:401-412.

26. PET imaging market summary report 2019. https://imvinfo.com/product/pet-imagingmarket-summary-report-2019. Accessed June 21, 2019.

27. Jack CR, Bennett DA, Blennow K, et al. NIA-AA research framework: toward a biological definition of Alzheimer's disease. Alzheimers Dement. 2018;14:535562

28. Seibyl JP, Kupsch A, Booij J, et al. Individual-reader diagnostic performance and between-reader agreement in assessment of subjects with parkinsonian syndrome or dementia using ${ }^{123}$ I-ioflupane injection (DaTscan) imaging. J Nucl Med. 2014; 55:1288-1296.

29. Tillisch J, Brunken R, Marshall R, et al. Reversibility of cardiac wall-motion abnormalities predicted by positron tomography. $N$ Engl J Med. 1986;314:884-888.

30. Wahl RL, Jacene H, Kasamon Y, Lodge MA. From RECIST to PERCIST: evolving considerations for PET response criteria in solid tumors. $J$ Nucl Med. 2009;50(suppl):122S-150S

31. Burggraaff $\mathrm{CN}$, Cornelisse AC, Hoekstra OS, et al. Interobserver agreement of interim and end-of-treatment ${ }^{18} \mathrm{~F}$-FDG PET/CT in diffuse large B-cell lymphoma: impact on clinical practice and trials. $J$ Nucl Med. 2018;59:1831-1836.

32. Aide N, Hicks RJ, Le Tourneau C, Lheureux S, Fanti S, Lopci E. FDG PET/CT for assessing tumour response to immunotherapy. Eur J Nucl Med Mol Imaging. 2019;46:238-250.

33. Mayer AT, Gambhir SS. The immunoimaging toolbox. J Nucl Med. 2018;59:11741182.

34. Su H, Bodenstein C, Dumont RA, et al. Monitoring tumor glucose utilization by positron emission tomography for the prediction of treatment response to epidermal growth factor receptor kinase inhibitors. Clin Cancer Res. 2006;12:5659-5667.

35. Mai WX, Gosa L, Daniels VW, et al. Cytoplasmic p53 couples oncogene-driven glucose metabolism to apoptosis and is a therapeutic target in glioblastoma. Nat Med. 2017;23:1342-1351.

36. Clark PM, Ebiana VA, Gosa L, Cloughesy TF, Nathanson DA. Harnessing preclinical molecular imaging to inform advances in personalized cancer medicine. J Nucl Med. 2017;58:689-696.
37. Lordick F, Ott K, Krause B-J, et al. PET to assess early metabolic response and to guide treatment of adenocarcinoma of the oesophagogastric junction: the MUNICON phase II trial. Lancet Oncol. 2007;8:797-805.

38. Benz MR, Czernin J, Allen-Auerbach MS, et al. FDG-PET/CT imaging predicts histopathologic treatment responses after the initial cycle of neoadjuvant chemotherapy in high-grade soft-tissue sarcomas. Clin Cancer Res. 2009;15:28562863.

39. Benz MR, Herrmann K, Walter F, et al. ${ }^{18} \mathrm{~F}-\mathrm{FDG}$ PET/CT for monitoring treatment responses to the epidermal growth factor receptor inhibitor erlotinib. $\mathrm{J} \mathrm{Nucl}$ Med. 2011;52:1684-1689.

40. Hertz B. A tribute to Dr. Saul Hertz: the discovery of the medical uses of radioiodine. World J Nucl Med. 2019;18:8-12.

41. Handkiewicz-Junak D, Poeppel TD, Bodei L, et al. EANM guidelines for radionuclide therapy of bone metastases with beta-emitting radionuclides. Eur J Nucl Med Mol Imaging. 2018;45:846-859.

42. Parker C, Nilsson S, Heinrich D, et al. Alpha emitter radium-223 and survival in metastatic prostate cancer. N Engl J Med. 2013;369:213-223.

43. Damm R, Seidensticker R, Ulrich G, et al. Y90 radioembolization in chemorefractory metastastic, liver dominant colorectal cancer patients: outcome assessment applying a predictive scoring system. BMC Cancer. 2016;16:509.

44. Wahl RL, Czernin J. Discussions with leaders: a conversation between Richard Wahl and Johannes Czernin. J Nucl Med. 2019;60:3-6.

45. Parent EE, Schuster DM. Update on ${ }^{18} \mathrm{~F}$-fluciclovine PET for prostate cancer imaging. J Nucl Med. 2018;59:733-739.

46. Ceci F, Castellucci P, Mapelli P, Incerti E, Picchio M, Fanti S. Evaluation of prostate cancer with ${ }^{11} \mathrm{C}$-choline $\mathrm{PET} / \mathrm{CT}$ for treatment planning, response assessment, and prognosis. J Nucl Med. 2016;57(suppl):49S-54S.

47. Bodei L, Ambrosini V, Herrmann K, Modlin I. Current concepts in ${ }^{68}$ Ga-DOTATATE imaging of neuroendocrine neoplasms: interpretation, biodistribution, dosimetry, and molecular strategies. J Nucl Med. 2017;58:1718-1726.

48. Graham MM, Gu X, Ginader T, Breheny P, Sunderland JJ. ${ }^{68}$ Ga-DOTATOC imaging of neuroendocrine tumors: a systematic review and metaanalysis. $\mathrm{J} \mathrm{Nucl}$ Med. 2017;58:1452-1458.

49. Morris E, Chalkidou A, Hammers A, Peacock J, Summers J, Keevil S. Diagnostic accuracy of ${ }^{18} \mathrm{~F}$ amyloid PET tracers for the diagnosis of Alzheimer's disease: a systematic review and meta-analysis. Eur J Nucl Med Mol Imaging. 2016;43:374-385.

50. Dani M, Brooks DJ, Edison P. Tau imaging in neurodegenerative diseases. Eur J Nucl Med Mol Imaging. 2016;43:1139-1150.

51. O'Keefe DS, Bacich DJ, Huang SS, Heston WDW. A perspective on the evolving story of PSMA biology, PSMA-based imaging, and endoradiotherapeutic strategies. J Nucl Med. 2018;59:1007-1013.

52. Hope TA, Goodman JZ, Allen IE, Calais J, Fendler WP, Carroll PR. Meta-analysis of ${ }^{68} \mathrm{Ga}$-PSMA-11 PET accuracy for the detection of prostate cancer validated by histopathology. J Nucl Med. 2019;60:786-793.

53. Giesel FL, Knorr K, Spohn F, et al. Detection efficacy of ${ }^{18}$ F-PSMA-1007 PET/ $\mathrm{CT}$ in 251 patients with biochemical recurrence of prostate cancer after radical prostatectomy. J Nucl Med. 2019;60:362-368.

54. Yin Y, Werner RA, Higuchi T, et al. Follow-up of lesions with equivocal radiotracer uptake on PSMA-targeted PET in patients with prostate cancer: predictive values of the PSMA-RADS-3A and PSMA-RADS-3B categories. $\mathrm{J} \mathrm{Nucl} \mathrm{Med}$. 2019;60:511-516.

55. Schmuck S, von Klot CA, Henkenberens C, et al. Initial experience with volumetric ${ }^{68} \mathrm{Ga}$-PSMA I\&T PET/CT for assessment of whole-body tumor burden as a quantitative imaging biomarker in patients with prostate cancer. $\mathrm{J}$ Nucl Med. 2017;58:1962-1968.

56. Tinkle CL, Duncan EC, Doubrovin M, et al. Evaluation of ${ }^{11} \mathrm{C}$-methionine PET and anatomic MRI associations in diffuse intrinsic pontine glioma. $\mathrm{J} \mathrm{Nucl} \mathrm{Med}$. 2019;60:312-319.

57. Wester HJ, Herz M, Weber W, et al. Synthesis and radiopharmacology of $O$-(2$\left[{ }^{18}\right.$ F]fluoroethyl)-L-tyrosine for tumor imaging. J Nucl Med. 1999;40:205-212.

58. Jager PL, Chirakal R, Marriott CJ, Brouwers AH, Koopmans KP, Gulenchyn KY. 6-L- ${ }^{18} \mathrm{~F}$-fluorodihydroxyphenylalanine PET in neuroendocrine tumors: basic aspects and emerging clinical applications. J Nucl Med. 2008;49:573-586.

59. Schwarzenberg J, Czernin J, Cloughesy TF, et al. Treatment response evaluation using ${ }^{18} \mathrm{~F}$-FDOPA PET in patients with recurrent malignant glioma on bevacizumab therapy. Clin Cancer Res. 2014;20:3550-3559.

60. Czernin J, Eiber M. Acceleration of PSMA-targeted theranostics to the clinic: can common sense prevail? J Nucl Med. 2017;58:1186-1187.

61. Reubi JC, Maecke HR. Approaches to multireceptor targeting: hybrid radioligands, radioligand cocktails, and sequential radioligand applications. J Nucl Med. 2017; 58(suppl):10S-16S.

62. Hope TA, Calais J, Zhang L, Dieckmann W, Millo C. ${ }^{111}$ In-pentetreotide scintigraphy vs. ${ }^{68} \mathrm{Ga}$-DOTATATE PET: impact on Krenning scores and effect of tumor burden. J Nucl Med. March 8, 2019 [Epub ahead of print] 
63. Hofman MS, Violet J, Hicks RJ, et al. [ $\left.{ }^{177} \mathrm{Lu}\right]-P S M A-617$ radionuclide treatment in patients with metastatic castration-resistant prostate cancer (LuPSMA trial): a single-centre, single-arm, phase 2 study. Lancet Oncol. 2018;19:825-833.

64. Fox JJ, Gavane SC, Blanc-Autran E, et al. Positron emission tomography/computed tomography-based assessments of androgen receptor expression and glycolytic activity as a prognostic biomarker for metastatic castration-resistant prostate cancer. JAMA Oncol. 2018;4:217-224.

65. Evans MJ, Smith-Jones PM, Wongvipat J, et al. Noninvasive measurement of androgen receptor signaling with a positron-emitting radiopharmaceutical that targets prostate-specific membrane antigen. Proc Natl Acad Sci USA. 2011;108: 9578-9582.

66. Dinan MA, Curtis LH, Hammill BG, et al. Changes in the use and costs of diagnostic imaging among Medicare beneficiaries with cancer, 1999-2006. JAMA. 2010; 303:1625-1631.

67. Yang Y, Czernin J. Contribution of imaging to cancer care costs. J Nucl Med. 2011;52(suppl):86S-92S.

68. Lynch TJ, Bell DW, Sordella R, et al. Activating mutations in the epidermal growth factor receptor underlying responsiveness of non-small-cell lung cancer to gefitinib. N Engl J Med. 2004;350:2129-2139.

69. Druker BJ, Talpaz M, Resta DJ, et al. Efficacy and safety of a specific inhibitor of the BCR-ABL tyrosine kinase in chronic myeloid leukemia. N Engl J Med. 2001; 344:1031-1037.

70. Sosman JA, Kim KB, Schuchter L, et al. Survival in BRAF V600-mutant advanced melanoma treated with vemurafenib. N Engl J Med. 2012;366:707-714.

71. Brower V. NCI-MATCH pairs tumor mutations with matching drugs. Nat Biotechnol. 2015;33:790.

72. Letai A. Functional precision cancer medicine: moving beyond pure genomics. Nat Med. 2017;23:1028.

73. Le Tourneau C, Delord J-P, Gonçalves A, et al. Molecularly targeted therapy based on tumour molecular profiling versus conventional therapy for advanced cancer (SHIVA): a multicentre, open-label, proof-of-concept, randomised, controlled phase 2 trial. Lancet Oncol. 2015;16:1324-1334.

74. Afshar-Oromieh A, Haberkorn U, Zechmann C, et al. Repeated PSMA-targeting radioligand therapy of metastatic prostate cancer with ${ }^{131}$ I-MIP-1095. Eur J Nucl Med Mol Imaging. 2017;44:950-959.
75. Kratochwil C, Bruchertseifer F, Rathke H, et al. Targeted $\alpha$-therapy of metastatic castration-resistant prostate cancer with ${ }^{225}$ Ac-PSMA-617: dosimetry estimate and empiric dose finding. J Nucl Med. 2017;58:1624-1631.

76. Werner RA, Bundschuh RA, Bundschuh L, et al. Interobserver agreement for the standardized reporting system PSMA-RADS 1.0 on ${ }^{18} \mathrm{~F}$-DCFPyL PET/CT imaging. J Nucl Med. 2018;59:1857-1864.

77. Fendler WP, Calais J, Eiber M, et al. Assessment of ${ }^{68}$ Ga-PSMA-11 PET accuracy in localizing recurrent prostate cancer: a prospective single-arm clinical trial. JAMA Oncol. March 28, 2019 [Epub ahead of print].

78. Loktev A, Lindner T, Burger EM, et al. Development of novel FAP-targeted radiotracers with improved tumor retention. J Nucl Med. March 8, 2019 [Epub ahead of print].

79. Lindner T, Loktev A, Altmann A, et al. Development of quinoline-based theranostic ligands for the targeting of fibroblast activation protein. J Nucl Med. 2018; 59:1415-1422.

80. Giesel FL, Kratochwil C, Lindner T, et al. ${ }^{68}$ Ga-FAPI PET/CT: biodistribution and preliminary dosimetry estimate of 2 DOTA-containing FAP-targeting agents in patients with various cancers. J Nucl Med. 2019;60:386-392.

81. Loktev A, Lindner T, Mier W, et al. A tumor-imaging method targeting cancerassociated fibroblasts. J Nucl Med. 2018;59:1423-1429.

82. Cronin KA, Lake A, Scott S, et al. Annual report to the nation on the status of cancer, part I: national cancer statistics. Cancer. 2018;124:2785-2800.

83. Weiner AB, Matulewicz RS, Schaeffer EM, Liauw SL, Feinglass JM, Eggener SE. Contemporary management of men with high-risk localized prostate cancer in the United States. Prostate Cancer Prostatic Dis. 2017;20:283-288.

84. Calais J, Czernin J, Cao M, et al. ${ }^{68} \mathrm{Ga}$-PSMA-11 PET/CT mapping of prostate cancer biochemical recurrence after radical prostatectomy in 270 patients with a PSA level of less than $1.0 \mathrm{ng} / \mathrm{mL}$ : impact on salvage radiotherapy planning. J Nucl Med. 2018;59:230-237.

85. Schöder H, Yeung HW, Larson SM. CT in PET/CT: essential features of interpretation. J Nucl Med. 2005;46:1249-1251.

86. International Atomic Energy Agency. Nuclear Medicine Resources Manual; Vienna, Austria: International Atomic Energy Agency; 2006.

87. Muylle K, Maffioli L. Nuclear medicine training in Europe: "all for one, one for all." J Nucl Med. 2017;58:1904-1905. 